\title{
The temperature characteristics of plasma induced by femtosecond laser radiation
}

\author{
Sergei Arakelyan, Svetlana Zhirnova, Arkady Galkin, Dmitriy Kochuev, \\ Elena Shamanskaya, and Kirill Khorkov \\ Vladimir State University, 600000 Vladimir, Russia
}

\begin{abstract}
In this work, the plasma temperature in an argon and nitrogen medium is measured at various pressure values. Such data can be used to calculate plasma parameters: electron concentration, collision frequency with atoms and molecules, etc. Two approaches are used to measure temperature: using a pyrometer and measuring the relative intensity of spectral lines.
\end{abstract}

As shown in [1,2], energy transported along an electric arc is used to increase the efficiency of obtaining nanoparticles upon ablation from the surface under the influence of laser radiation. In this case, an effective correction of the electric arc can be carried out using optical discharge (OD). To optimize the OD, it is necessary, in particular, to know the characteristics of the OD plasma, such as the electron concentration, plasma temperature, and its conductivity.

The electron temperature of the laser-induced plasma channels in argon and nitrogen media was measured at various pressures from the relative intensities of the spectral lines according to the following scheme. The laser radiation of the femtosecond laser system was focused into the internal volume of the vessel using a focusing lens with a focal length of 150 $\mathrm{mm}$; an optical breakdown is formed in the focal plane of the focusing lens. The geometric characteristics and brightness of the glow were recorded by a CCD camera. The volume of the vessel was filled from a cylinder containing the necessary gas, the pressure in the vessel was controlled by a reducer equipped with shut-off and measuring equipment. The spectrum of the output radiation was studied using a spectrometer. The data from the spectrometer and CCD camera during the experimental work was collected on a personal computer. Plasma spectra were obtained at various pressure values.

The obtained spectral lines were recorded at an exposure of $2 \mathrm{sec}$. A long exposure time is necessary to obtain a good response of the spectrometer matrix and to average the recorded signal. The parameters for recording spectra are similar for all experiments.

The electron temperature was calculated in accordance with [3]. The final formula for calculating the electron temperature is:

$$
\mathrm{T}_{\mathrm{e}}=\frac{-\Delta E}{\left(\ln \left(\frac{I_{2}}{I_{1}}\right)-\ln \left(\frac{g_{2} f_{2}}{g_{1} f_{1}} *\left(\frac{\lambda_{1}}{\lambda 2}\right)^{3}\right)\right) * k},
$$

$\lambda_{1}$ and $\lambda_{2}$ - the wavelength of the spectral line (nm) for calculations it is more convenient to use the most spaced pairs of lines. For argon, the lines of $415,85 \mathrm{~nm}$ and $852,14 \mathrm{~nm}$ were 
used; for nitrogen, the lines were $746,83 \mathrm{~nm}$ and $493,5 \mathrm{~nm}$. The intensities of spectral lines I were obtained from the spectrometer.

$\Delta E$ - the difference between the excitation energies of the studied lines ; $f$ - the oscillator strength; $g$ - the statistical weight of the level $; \lambda$ - the wavelength of the corresponding spectrum line, $k$ - Boltzmann constant.

The experiments on measuring the electronic temperature of laser-induced plasma channels in argon and nitrogen media at various pressures by the photopyrometry method were carried out using a pulsed laser system, pulse duration $280 \mathrm{fs}$, pulse repetition rate 10 $\mathrm{kHz}$, pulse energy $150 \mu \mathrm{J}$, emission wavelength $1030 \mathrm{~nm}$. Laser radiation was focused by a single plane-convex lens with a focal length of $150 \mathrm{~mm}$ into a vessel, which makes it possible to study laser-induced processes in various media at different pressures and temperatures.

When measuring the temperature of a laser-induced plasma in a gas (argon and nitrogen) at different pressures, the MP-1001 high-temperature micropyrometer was used. This device allows you to measure the brightness temperature in the range from 1500 to $5000 \mathrm{~K}$. The transition from brightness temperature $\left(T_{b}\right)$ to true $\left(T_{t}\right)$ is carried out in accordance with the formula [4]:

$$
\mathrm{T}_{t}=\frac{T_{b} \cdot C_{2}}{\mathrm{C}_{2}+\lambda \mathrm{T}_{b} \cdot \ln \alpha}
$$

$\lambda=650 \mathrm{~nm}$ is the reference / calibrated won length ; $C_{2}=0,014388 \mathrm{~m} \cdot \mathrm{K}-$ the second Planck constant, $\alpha$ - the degree of blackness (absorption coefficient). The degree of blackness was determined by measuring the intensity of the transmitted reference / probe laser beam with a wavelength of $650 \mathrm{~nm}$ through the volume of the laser-induced plasma channel.

When considering the graphs of the dependence of the temperature of the plasma channels on the pressure obtained using two different methods, an increase in temperature with an increase in pressure is observed. As for the comparison of methods, the temperature data obtained in two different ways are related to each other, the difference in values is small, and is not critical (does not exceed 10\%).

The plasma temperature measured by both methods was in the range of $\sim 4000-5000 \mathrm{~K}$, depending on the pressure. For nitrogen with a monotonic increase in the range of indicated temperatures from 0 to a pressure of $15 \mathrm{bar}$, and for argon with a maximum at pressures of about 7-9 bar.

Since, according to the corresponding estimates, the characteristic heat transfer time of electrons with heavy particles is fractions of a microsecond (quick relaxation), and the interpulse period is $100 \mu \mathrm{s}$, the electron temperature actually determined during the measurement is equal to the gas temperature, that is, we are dealing with local thermodynamics equilibrium.

\section{References}

1. A.V. Ivashenko, D.A. Koshuev, R.V.Chkalov, K.S. Khorkov, J. Phys.: Conf. Ser. IOP Publishing 1164, 012021, (2019).

2. A.V. Ivashenko, D.A. Koshuev, R.V. Chkalov, LIEES Mat. Today: Proc. 11, 465-470 (2019).

3. S.Yu. Lukyanov, Hot plasma and controlled nuclear fusion (Moscow, Nauka, 1975).

4. A.N. Magunov, Scien. Instr. 20, 22-26 (2010). 